\title{
The need for biokineticists in the South African public health care system
}

\author{
R W Evans, ${ }^{1}$ MPhil, T Smith, ${ }^{1}$ BA, P Kay, ${ }^{1}$ MPhil, D McWade, ${ }^{1}$ MPhil, N Angouras, ${ }^{1}$ BSc (Hons), R F van Aarde, ${ }^{1}$ MPhil, R Arkell, \\ ${ }^{1}$ MPhil, E V Lambert, ${ }^{1} \mathrm{PhD}, \mathbf{N}$ van der Schyff, ${ }^{2} \mathrm{MD}$ \\ 1 Division of Exercise Science and Sports Medicine, Department of Human Biology, Faculty of Health Sciences, University of Cape Town, Cape Town, South \\ Africa \\ Victoria Hospital, Wynberg, Cape Town, South Africa
}

Corresponding author: $R$ W Evans (robertevanssa@gmail.com)

Background: Noncommunicable diseases (NCDs) are increasingly prevalent within South Africa. Physical inactivity is a significant, independent and modifiable risk factor increasing the prevalence of NCDs.

Discussion: The integration of physical activity programmes into the primary health care system through multidisciplinary platforms is thus advocated for and envisioned to be more costeffective than current practices. However, currently within the primary health care setting of South Africa, there is an absence of health care professionals adequately equipped to develop and implement physical activity programmes. Biokineticists, whose scope of practice is to improve physical functioning and health through exercise as a modality, are ideally suited to developing and implementing physical activity programmes in the public sector. Yet despite their evident demand, the role of the biokineticist is not incorporated into the national public health care system.

Conclusion: This short report calls firstly, for the inclusion of biokinetics into the public health care sector, and secondly, for the funding of multidisciplinary community health programmes supporting education, healthy eating and physical activity levels.

Keywords: noncommunicable disease, physical activity, community health programme, primary health care

S Afr J Sports Med 2016;28(3):85-86.DOI: 10.17159/2078-516X/2016/v28i3a1310

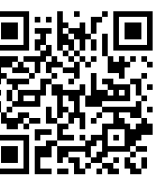

Noncommunicable diseases and physical inactivity

One in two South Africans are physically inactive $^{[1]}$, a statistic which urgently needs to be addressed and managed to curb the rampant increase in noncommunicable diseases (NCDs). NCDs are a category of chronic diseases that are not infectious and hence cannot be passed on from one person to another. They typically progress at a slow rate and include conditions such as cardiovascular disease, cancer, chronic respiratory diseases and diabetes mellitus. ${ }^{[2]}$

A South African Summit on the Prevention and Control of Noncommunicable Diseases was held on the 12-13 September 2011. The summit reached a consensus regarding the need for an intensified national action plan, addressing prevention, early detection, behavioural change and universal treatment of NCDs in South Africa. This consensus materialised in the form of the Strategic Plan for Prevention and Control of Noncommunicable Diseases 2013-2017. The strategy, developed by the Department of Health, identified three public health strategies. ${ }^{[3]}$

1. Prevent NCDs and promote health and wellness at population, community and individual levels,

2. Improve control of NCDs through the strengthening and reform of health care systems,
3. Monitor NCDs and their main risk factors, as well as the conducting of innovative research.

Physical inactivity is recognised internationally as a significant, independent and modifiable risk factor contributing to the increased prevalence of NCDs..$^{[4]}$ Joubert ${ }^{[1]}$ identified that, based on the 1998 South African Demographic and Health Survey data, an alarming number of NCDs amongst South Africans were attributable to physical inactivity. ${ }^{[5]}$ Physical inactivity was identified as attributable to $47 \%$ of ischemic heart diseases and $20 \%$ of patients with diabetes mellitus; $87 \%$ of cases of type 2 diabetes were attributable to excess body weight, along with $68 \%$ of hypertensive disease.

\section{Cost-effective approaches to combatting noncommunicable diseases}

The associated morbidity of health disorders related to inactivity, including health-related quality of life, as well as direct and indirect economic costs, exerts a substantial burden on societies and health systems. In South Africa, use of physicians to address physical inactivity has been estimated to cost R11.80 per head. When compared to the R4.50 per head required for worksite interventions, it is evident that the use of physicians is a relatively large expense.[3]

The Global Advocacy for Physical Activity (GAPA) describes seven best investments for physical activity, advocating for the integration of physical activity into the primary health care system to reduce the development of NCDs. ${ }^{[6]}$ The recommended approach to implementing a physical activity programme is a combination of brief counselling and links to community-based supports. These recommendations do not exclude the physician or other health care providers from the management of physical activity; rather it emphasises that a multidisciplinary approach is needed to reduce physical inactivity. Biokineticists, in conjunction with physicians and other practitioners, such as dietitians, physiotherapists and psychologists, are in the best position to contribute towards reducing physical inactivity.

Many biokinetics students are denied internship positions due to a lack of capacity within the private health care sector. The utilisation of both interns and qualified biokineticists in the public health care sector provides a cost-effective approach to combatting an increasingly sedentary South African lifestyle.

\section{Biokinetics in the South African public health care sector}

Lee $^{[7]}$ demonstrated that a decrease in physical inactivity by $10 \%$ will result in excess of 533000 lives saved due to NCDs around the world every year. Despite these statistics, South Africa is without a health care professional within the primary health care setting that is adequately equipped to develop and implement physical activity programmes. The primary function of a biokineticist is to improve physical functioning and health 
through exercise as a modality. It would thus appear that a biokineticist is ideally suited to developing and implementing programmes to reduce physical inactivity in the public sector. It is evident that based on the biokineticist's scope of practice; there is an overwhelming demand for their skills in combatting the current burden of NCDs facing the country.

The demand for biokineticists is evident within the private health care sector as demonstrated by Moss and Lubbe. ${ }^{[8]}$ However, the vast majority of people (40 million people) cannot afford the expense of private health care. They are therefore reliant on a public health care system already crippled by a quadruple burden of disease of HIV/AIDS and tuberculosis (TB), high maternal and child mortality, high levels of violence and injuries, and a growing burden of NCDs.

\section{Conclusion and recommendations}

South Africa is without a focused approach to reducing physical inactivity. The South African public health care sector needs to progress beyond treating NCDs and adopt strategies focused on prevention. Health promotion using physical activity falls directly within the biokineticist's scope of practice. ${ }^{[9]}$

This short report calls for the support and funding of community-based physical activity interventions in combatting NCDs. The time has come for South African policymakers to act upon the Strategic Plan for Prevention and Control of NCDs 2013-2017. These actions should be the inclusion of Biokinetics into the public health care sector and the funding of multidisciplinary community health programmes supporting education, healthy eating and physical activity levels.

\section{References}

1. Joubert J, Norman R, Lambert EV, et al. Estimating the burden of disease attributable to physical inactivity in South Africa in 2000. S Afr Med J 2007; 97(8 Pt 2):725-731. PMID: 17952230

2. American College of Sports Medicine. ACSM's guidelines for exercise testing and prescription. $9^{\text {th }}$ ed. Lippincott Williams \& Wilkins; 2013. doi:10.1249/jsr.0b013e31829a68cf

3. South Africa. Department of Health. Strategic Plan for the Prevention and Control of Non-Communicable Diseases 2013-17. Department of Health 2013. Available from: http://www.hsrc.ac.za/uploads/pageContent/3893/NCDs\%20STRAT\%20P LAN\%20\%20CONTENT\%208\%20april\%20proof.pdf

4. Steyn K, Fourie J, Temple N (eds). Chronic diseases of lifestyle in South Africa: 1995-2005. Technical Report. Cape Town: South African Medical Research Council, May 2006:33-47. Available from: http://www.mrc.ac.za/noncomm/cdl1995-2005.pdf

5. South Africa. Department of Health. South Africa Demographic and Health Survey 1998. Department of Health, 1998. Available from:http://www.mrc.ac.za/bod/SADHS1998FullReport.pdf

6. Global Advocacy for Physical Activity (GAPA) the Advocacy Council of the International Society for Physical Activity and Health (ISPAH). NCD Prevention: Investments that Work for Physical Activity. February 2011. Available from: http://www.globalpa.org.uk/pdf/investments-work.pdf

7. Lee IM, Shiroma EJ, Lobelo F, et al. Effect of physical inactivity on major non-communicable diseases worldwide: an analysis of burden of disease and life expectancy. Lancet 2012 Jul 27;380(9838):219-229.doi: 10.1016/S0140-6736(12)61031-9; PMID: 22818936

8. Moss SJ, Lubbe MS. The potential market demand for biokinetics in the private health care sector of South Africa. S Afr J Sports Med 2011;23(1):1419.

9. Health Professions Council of South Africa (HPCSA). Medical, Dental and Supplementary Health Service Professions Act (Act No. 56 of 1974), Section 33 (1), c1974 [cited 2016 May 18]. Available from http://www.hpcsa.co.za/Uploads/editor/UserFiles/downloads/rules_reg_c onstitution/regulations_defining_the_scope_of_the_profession_of_biokine tics.pdf 